\title{
1 The role of antifreeze glycopeptides (AFGP) and polyvinyl 2 alcohol/polyglycerol (X/Z-1000) cocktails as ice modulators 3 during partial freezing of rat livers.
}

4

3
Shannon N. Tessier ${ }^{1,2}$, Omar Haque ${ }^{1,2,3,4}$, Casie A. Pendexter ${ }^{1,2}$, Stephanie E.J. Cronin ${ }^{1,2}$, Lindong Weng ${ }^{1,2}$, Heidi Yeh ${ }^{4}$, James F. Markmann ${ }^{4}$, Michael J. Taylor ${ }^{5}$, Gregory M. Fahy ${ }^{6}$, Mehmet Toner ${ }^{1,2, *}$, Korkut Uygun ${ }^{1,2, *}$

${ }^{1}$ Center for Engineering in Medicine and Surgery, Massachusetts General Hospital, Harvard Medical School, Boston MA, USA

${ }^{2}$ Shriners Hospitals for Children - Boston MA, USA

${ }^{3}$ Department of Surgery, Beth Israel Deaconess Medical Center, Boston MA, USA

${ }^{4}$ Department of Surgery, Division of Transplantation, Massachusetts General Hospital, Harvard Medical School, Boston MA, USA

${ }^{5}$ Sylvatica Biotech Inc. North Charleston SC, USA and Department of Mechanical Engineering, Carnegie Mellon University, Pittsburgh, PA USA

621st Century Medicine, Fontana, California, USA

\section{*Co-Corresponding authors:}

Korkut Uygun

Tel: 617-371-4881

Email: KUYGUN@mgh.harvard.edu

Mehmet Toner

Tel: 617-724-5336

Email: mehmet_toner@hms.harvard.edu 
39 Abstract

The current liver organ shortage has pushed the field of liver transplantation to develop

41 new methods to prolong the preservation time of livers from the current clinical standard of static

42 cold storage. Our approach, termed partial freezing, aims to induce a thermodynamically stable

43 frozen state at deeper storage temperatures $\left(-10^{\circ} \mathrm{C}\right.$ to $\left.-15^{\circ} \mathrm{C}\right)$ than can be achieved with

44 supercooling, while simultaneously maintaining a sufficient unfrozen fraction to limit

45 dehydration and ice damage. This research first demonstrated that partially frozen glycerol

46 treated rat livers were functionally similar after thawing from either -10 or $-15^{\circ} \mathrm{C}$ with respect to

47 subnormothermic machine perfusion metrics and histology. Next, we assessed the effect of

48 adding either of two ice modulators, antifreeze glycoprotein (AFGP) and a polyvinyl

49 alcohol/polyglycerol combination (X/Z-1000), on the viability and structural integrity of partially

50 frozen rat livers compared to glycerol-only control livers. Results showed that AFGP livers had

51 high levels of ATP and the least edema but suffered from significant endothelial cell damage.

$52 \mathrm{X} / \mathrm{Z}-1000$ livers had the highest levels of ATP and energy charge (EC) but also demonstrated

53 endothelial damage and post-thaw edema. Glycerol-only control livers exhibited the least DNA

54 damage on Terminal deoxynucleotidyl transferase dUTP nick end labeling (TUNEL) staining but

55 also had the lowest levels of ATP and EC. Further research is necessary to optimize the ideal ice

56 modulator cocktail for our partial-freezing protocol. Modifications to cryoprotective agent (CPA)

57 combinations, as well as improvements to machine perfusion CPA loading and unloading, can

58 help improve the viability of these partially frozen organs.

60 Key words: antifreeze glycopeptides, polyvinyl alcohol, polyglycerol, cryopreservation,

61 partial freezing, subnormothermic machine perfusion 


\section{Abbreviations}

63 3-OMG, 3-O-methyl-D-glucose

64 ANOVA, Analysis of variance

65 AFGP, Antifreeze glycoprotein

66 BSA, Bovine serum albumin

67 CPA, Cryoprotective agent

68 EC, Energy charge

69 H\&E, Hematoxylin and Eosin

70 HES, Hydroxyethyl starch,

71 HLA, Human leukocyte antigen

72 HMP, Hypothermic machine perfusion

73 INAs, Ice nucleating agents

74 IRIs, Ice recrystallization inhibitors

75 IVC, Infrahepatic vena cava

76 IACUC, Institutional Animal Care and Use Committee

77 LT, Liver transplant

78 M, molarity

79 PEG, Polyethylene glycol

80 X/Z-1000, Polyvinyl alcohol/polyglycerol

81 PV, Poral Vein

82 SCS, Static cold storage

83 SNMP, Subnormothermic machine perfusion

84 TUNEL, Terminal deoxynucleotidyl transferase dUTP nick end labeling

85 UW, University of Wisconsin

86 WE, Williams E

87

88

89

90

91

92

93

94

95

96 


\section{I. Introduction}

The liver organ shortage has pushed the field of transplantation to develop bold new

99 strategies to preserve transplantable organs. Currently, the clinical standard of preserving

100 transplantable livers is static cold storage (SCS) at $4^{\circ} \mathrm{C}$, which keeps organs viable for a

101 maximum of 12 hours [1]. Prolonging this preservation time would improve the allocation of

102 organs in many ways. For example, it would reduce organ discard due to unacceptably long

103 ischemic times, lower operating room costs by making liver transplant (LT) operations elective,

104 enhance donor-recipient selection with human leukocyte antigen (HLA) matching and global

105 matching programs, and make tolerance induction protocols more feasible [2-4].

Preservation methods to slow organ deterioration after procurement can be broadly

107 categorized into two strategies: metabolic support and metabolic depression. Metabolic support

108 through ex-vivo machine perfusion allows for continuous quality and viability assessment of

109 organs. However, the major challenge with long term machine perfusion is maintaining organ

110 homeostasis ex-vivo, which becomes exponentially more complex with longer perfusion

111 durations that require continuous monitoring and adaptations [3,5-8]. On the other hand,

112 metabolic depression strategies leverage the fact that tissue deterioration slows down at

113 decreasing temperatures. Furthermore, lowering the hypothermic preservation temperature below

$1144^{\circ} \mathrm{C}$ harbors great potential to extend preservation times beyond clinical standards and does not

115 require the long-term, constant maintenance of machine perfusion [9].

116 Within metabolic depression preservation strategies, most subzero preservation efforts

117 have centered on low cryogenic temperature ranges $\left(<-80^{\circ} \mathrm{C}\right)$ [3]. However, recent work has

118 investigated prospects for expanding storage within the high subzero temperature range from -

$1194^{\circ} \mathrm{C}$ to $-20^{\circ} \mathrm{C}$. These temperatures allow for more metabolic depression than hypothermic SCS 
120 while also potentially avoiding lethal ice formation and vitrification-related cryoprotectant

121 toxicity and thermal stresses. The most prominent present example of the potential of this

122 approach has involved storage below the thermodynamic freezing point at $-4^{\circ} \mathrm{C}$ to $-6^{\circ} \mathrm{C}$ in the

123 absence of ice (termed supercooling), which has enabled 3-fold extensions of liver preservation

124 duration [9-11]. While these studies have shown that ice formation in rodent and human livers

125 can be completely circumvented with supercooling $[9,11,12]$, the depth of metabolic stasis that

126 can be achieved by this method is limited by the risk of spontaneous nucleation leading to

127 damaging ice formation, which increases as temperature is lowered to below $-6^{\circ} \mathrm{C}[13]$.

Although there is extensive evidence that ice formation can be severely damaging in

129 tissues and organs [14-17], recent studies based on the survival of frozen animals such as frogs

130 and turtles in nature have suggested that carefully limited and controlled ice formation may be

131 tolerable during attempted cryopreservation of solid organs [2,18]. Ishine et al. showed that ice

132 modulators such as antifreeze glycoproteins (AFGP) can have protective effects in high subzero

133 liver freezing protocols by inhibiting ice recrystallization and preventing ionic leakage through

134 cell membranes at low temperatures, although the authors report damage to the endothelial layer.

135 The authors froze their rat livers for 2 hours used livers frozen with glycerol as the only

136 cryoprotective agent (CPA) as controls [19]. To expand on these efforts, we aimed to test

137 extended preservation durations (up to 5 days) in the presence of two ice modulators, antifreeze

138 glycoprotein (AFGP) and a polyvinyl alcohol/polyglycerol combination (X/Z-1000), for their

139 ability to confer freeze tolerance of rodent livers. The inclusion of these agents is called for in

140 part because although the total quantity of ice present during long term storage at a fixed

141 temperature is constant, the ice may still cause injury due to recrystallization that could be

142 overcome by ice modulators. 
AFGP has been shown to inhibit both ice recrystallization and ice growth below $\mathrm{T}_{\mathrm{M}}$ (the

144 thermodynamic freezing point). These glycopeptides inhibit ice growth by attaching to multiple

145 faces of ice crystals [20-23]. AFGPs have also been shown to raise the homogenous ice

146 nucleation temperature $\left(\mathrm{T}_{\mathrm{H}}\right)$ by organizing water into a more ice-like state [24]. However, since

147 the temperature range in our partial freezing protocol is well above $T_{H}[25]$, the issues at hand

148 involve the role of AFGP in ice shaping and ice recrystallization inhibition. Although AFGP can

149 shape ice into damaging spicules [26], this effect may be outweighed under our storage

150 conditions by the ice growth and recrystallization inhibitory effects of AFGP. X-1000 is a 2

151 kilodalton ( $\mathrm{kDa}$ ) polyvinyl alcohol [27] that contains 20\% vinyl acetate, which improves the

152 solubility and ice-inhibiting effects of X-1000 presumably by preventing self-association

153 between X-1000 chains. Polyvinyl alcohol is known to inhibit ice recrystallization [28,29]. Z-

1541000 is a polyglycerol that inhibits heterogeneous ice nucleation [30], and together X/Z-1000 has

155 been shown to protect rat hearts during supercooling [31-33] and is functional from $0^{\circ} \mathrm{C}$ to

156 temperatures below $-120^{\circ} \mathrm{C}[16]$.

157 In the present study, whole rat livers were frozen for up to 5 days at high subzero

158 temperatures $\left(-10^{\circ} \mathrm{C}\right.$ to $\left.-15^{\circ} \mathrm{C}\right)$ by combining glycerol and ice nucleating agents (INAs) with the

159 use of subnormothermic machine perfusion (SNMP) at $21^{\circ} \mathrm{C}$. Further, two ice modulators,

160 antifreeze glycoprotein (AFGP) and a polyvinyl alcohol/polyglycerol combination (X/Z-1000),

161 were tested. Livers frozen with the inclusion of either AFGP or X/Z-1000 were compared to the

162 control group (with glycerol as the main CPA) with primary outcomes being perfusion metrics,

163 ATP, energy charge (EC), weight gain, and histology. We call this protocol "partial freezing"

164 since it induces a thermodynamically stable frozen state at deeper storage temperatures (as low 
as $-15^{\circ} \mathrm{C}$ ) than can be achieved with supercooling, while simultaneously maintaining a sufficient unfrozen fraction to limit dehydration and ice damage.

\section{Materials and Methods}

\section{A. Experimental design}

Figure 1 outlines the rat liver partial freezing protocol in 8 consecutive steps: (1) liver

171 procurement, (2) preconditioning during SNMP, (3) CPA preloading during hypothermic

172 machine perfusion (HMP), (4) loading of final storage solution and ice modulators during HMP,

173 (5) partial freezing of rat liver, (6) thawing of rat liver, (7) unloading of CPAs during HMP, and

174 (8) functional recovery of frozen rat livers during SNMP.

175 Fig 1. Experimental design of partial freezing, in 8 consecutive steps. (1) liver

176 procurement, (2) preconditioning during SNMP, (3) CPA preloading during hypothermic

177 machine perfusion (HMP), (4) loading of final storage solution and ice modulators during HMP,

178 (5) partial freezing of rat liver, (6) thawing of rat liver, (7) unloading of CPAs during HMP, and

179 (8) functional recovery of frozen rat livers during SNMP.

180 Within this protocol, we first compared partially frozen livers at $-10^{\circ} \mathrm{C}(\mathrm{n}=4$ livers $)$ and -

$18115^{\circ} \mathrm{C}$ ( $\mathrm{n}=9$ livers) with $12 \%$ glycerol. Upon finding minimal differences between these two

182 groups, we combined them as a control and compared them to livers partially frozen with 0.5

$\mathrm{mg} / \mathrm{ml}(0.05 \% \mathrm{w} / \mathrm{v})$ of AFGP $(\mathrm{n}=4$ livers $)$ or $0.1 \% \mathrm{X}-1000 / 0.2 \% \mathrm{Z}-1000($ total, $0.3 \% \mathrm{w} / \mathrm{v} ; \mathrm{n}=4$

184 livers) ice modulating agents added to the preservation solution. After freezing, liver viability on

185 SNMP was compared between the $12 \%$ glycerol control group and the two ice modulated

186 groups. 


\section{B. Partial freezing protocol}

The rat liver perfusion system involved perfusion through the cannulated portal vein (PV)

with regulation of pressure, flow, and temperature. Detailed set-up of the perfusion system has

been previously described [34]. The total rat hepatectomy protocol was approved by the

193 (Boston, MA, USA). Livers were procured from male Lewis rats (250-300g, age 10-12 weeks.

194 Charles River Laboratories, Wilmington, MA, USA) (figure 1, step 1). The bile duct was

195 isolated and cannulated, and the rats were heparinized with $30 \mathrm{U}$ sodium heparin. PV splenic and

196 gastric branches, as well as the hepatic artery were ligated prior to cannulation. The PV was

197 subsequently cannulated with a 16-gauge catheter and immediately flushed with 40ml of

198 heparinized saline (1000U/ml at room temperature). The liver was then freed from all peritoneal 199 attachments, flushed with an additional $20 \mathrm{ml}$ of heparinized saline. After procurement, the liver 200 was weighed, and machine perfusion was immediately initiated.

201 Preconditioning during SNMP was initiated at $21^{\circ} \mathrm{C}$ with a flow rate of $5 \mathrm{ml} / \mathrm{min}$ (figure

202 1, step 2). The flow rate was gradually increased $[(1 \mathrm{ml} / \mathrm{min}) / \mathrm{min}]$ until a maximum PV pressure

203 of $5 \mathrm{mmHg}$, or a flow rate of $25 \mathrm{ml} / \mathrm{min}$, was reached (whichever was reached first). The rat

204 livers were perfused for 1 hour with $500 \mathrm{ml}$ of preconditioning solution consisting of Williams E, $205200 \mathrm{U} / 1$ of insulin, 2\% w/v PEG, 50g/L BSA, 100mM 3-O-methylglucose (3-OMG), 10,000 U/1

206 of heparin, $24 \mathrm{mg} / \mathrm{L}$ of dexamethasone, $25 \mathrm{mg} / \mathrm{ml}$ of hydrocortisone, 40,000 ug/l of penicillin, 207 and 40,000 U/1 of streptomycin and sodium bicarbonate as needed to maintain a physiological $208 \mathrm{pH}$ (see Supplemental Table 1 for composition of all solutions). 
After 1 hour of preconditioning during SNMP, the temperature was decreased to $4^{\circ} \mathrm{C}$ at a

210 rate of $\sim 1^{\circ} \mathrm{C} / \mathrm{min}$. Flow rates were gradually adjusted as necessary to ensure a maximum

211 perfusion pressure of 3-5mmHg during HMP. The SNMP preconditioning solution was switched

212 to $500 \mathrm{ml} \mathrm{CPA} \mathrm{preloading} \mathrm{solution} \mathrm{at} 4^{\circ} \mathrm{C}$ (consisting of Williams E, $200 \mathrm{U} / 1$ of insulin, $2 \%$

213 PEG, 50g/L BSA, 100mM 3-OMG, 30mM raffinose, 3\% hydroxyethyl starch (HES), 6\%

214 glycerol, $4000 \mathrm{U} / 1$ of heparin, $24 \mathrm{mg} / 1$ of dexamethasone, $25 \mathrm{mg} / \mathrm{ml}$ of hydrocortisone, 40,000

$215 \mathrm{ug} / \mathrm{l}$ of penicillin, 40,000 U/1 of streptomycin, and sodium bicarbonate as needed to maintain a

216 physiological $\mathrm{pH}$ ) (figure 1, step 3; Table S1). HMP was continued for 1 hour to ensure

217 complete equilibration of solution throughout the liver parenchyma.

After CPA preloading during HMP, rat livers were loaded with 50mls of final storage

219 solution (consisting of University of Wisconsin (UW) Solution (Bridge to Life Ltd., Columbia,

$220 \mathrm{SC}, \mathrm{USA}$ ), 5\% PEG, 50mM trehalose, 12\% v/v (1.64M) glycerol, 1g/L Snomax (Telemet,

221 Hunter, NY, USA) to promote nucleation, $10 \mathrm{U} / 1$ of insulin, $24 \mathrm{mg} / \mathrm{l}$ of dexamethasone, sodium

222 bicarbonate as needed to maintain a physiological $\mathrm{pH}$, and either $0.5 \mathrm{mg} / \mathrm{ml}$ of AFGP or $0.1 \% \mathrm{x}$ -

$2231000 / 0.2 \%$ z-1000 (figure 1, step 4). During the perfusion of the final storage solution the liver

224 was perfused at a fixed flow rate of $0.5 \mathrm{ml} / \mathrm{min}$ for $30 \mathrm{~min}$, resulting in a perfusion pressure of 3

$225 \mathrm{mmHg}$.

226 Once the final storage solution during HMP had been perfused through the liver, the

227 livers were placed in a storage bag with $50 \mathrm{ml}$ of storage solution in a pre-cooled chiller (Engel,

228 Schwertberg, Austria; model no. ENG65-B) for partial freezing (figure 1, step 5). Note: the

229 concentrations of raffinose and HES were higher in the storage solution than in the pre-loading

230 solution because the storage solution was made with UW which contains both raffinose and

231 HES. In the case of livers frozen with glycerol only, the chiller temperature was pre-cooled to 
232 either $-10^{\circ} \mathrm{C}$ or $-15^{\circ} \mathrm{C}$, and the liver was stored at one of these temperatures for 1 to 5 days.

233 Livers frozen with either of the two ice modulation candidates were stored at $-15^{\circ} \mathrm{C}$.

235 thawing solution consisting of Williams E, $10 \mathrm{U} / 1$ of insulin, 2\% PEG, 50g/L BSA, 100mM 3-

$236 \mathrm{OMG}, 30 \mathrm{mM}$ raffinose, 3\% HES, 50mM trehalose, $5 \mathrm{mM}$ L-glutathione, $200 \mu \mathrm{M}$ cyclic AMP,

$2371,000 \mathrm{U} / \mathrm{l}$ of heparin, $24 \mathrm{mg} / \mathrm{l}$ of dexamethasone, $25 \mathrm{mg} / \mathrm{ml}$ of hydrocortisone, $40,000 \mathrm{ug} / \mathrm{l} \mathrm{of}$

238 penicillin, and 40,000 U/1 of streptomycin, which was pre-warmed to $37^{\circ} \mathrm{C}$ in a constant

239 temperature bath (Thermo Fisher, Waltham, MA, USA). Livers were gently agitated until fully

240 thawed, which required approximately $5 \mathrm{~min}$ based on superficial observation and thermal

241 equilibration between the final thawing solution temperature and the liver surface temperature of

$2424^{\circ} \mathrm{C}$.

243

After thawing, CPAs and INAs were unloaded during HMP (figure 1, step 7). The

244 thawed livers were perfused at $4^{\circ} \mathrm{C}$ with thawing solution for 60 min with an initial flow rate of 2

$245 \mathrm{ml} / \mathrm{min}$. Flows were increased so as to keep a maximum pressure of $3 \mathrm{mmHg}$. After $60 \mathrm{~min}$, the

246 perfusion temperature was increased to $21^{\circ} \mathrm{C}$, and the perfusion solution was changed to $750 \mathrm{ml}$

247 of SNMP recovery solution (consisting of Williams E, $20 \mathrm{U} / 1$ of insulin, 2\% PEG, 50g/L BSA,

$2485 \mathrm{mM}$ L-glutathione, 200uM cyclic AMP, 1,000 U/1 of heparin, $24 \mathrm{mg} / \mathrm{l}$ of dexamethasone, 25

$249 \mathrm{mg} / \mathrm{ml}$ of hydrocortisone, $40000 \mathrm{ug} / 1$ of penicillin, and $40000 \mathrm{U} / 1$ of streptomycin; Table S1),

250 discarding the first 50-100mls to ensure complete CPA removal. Functional recovery of frozen

251 rat livers during SNMP with recovery solution continued for 3 hours (figure 1, step 8) with a

252 maximum intrahepatic perfusion pressure of $5 \mathrm{mmHg}$ and a maximum flow rate of $25 \mathrm{ml} / \mathrm{min}$. 
Perfusate measurements were done hourly during the SNMP recovery period. Time zero

$256(\mathrm{t}=0)$ was defined as being at approximately 5 min of HMP, and the first outflow samples were

257 taken at this time (flow, $2 \mathrm{ml} / \mathrm{min}$ ). PV and infrahepatic vena cava (IVC) oxygen partial

258 pressures and lactate levels were measured with a Cg4+ i-STAT cartridge (catalog no. 03P85-50)

259 and handheld blood analyzer. Similarly, potassium and other electrolytes were measured in IVC

260 samples using a Chem 8+ i-STAT cartridge (catalog no. 09P31-26) with the same blood analyzer

261 (catalog no. WD7POC012; Abbott, Chicago, IL, USA).

Rat liver weight was measured directly after procurement, prior to freezing, after

263 thawing, and after viability testing. Weight gain was calculated as the percentage increase at the

264 end of recovery compared to the liver weight after procurement. Vascular resistance was

265 calculated by dividing the perfusion pressure in the PV by the flow rate per gram of liver using

266 the weight of the liver after procurement as the reference standard weight. Oxygen consumption

267 rates were calculated as $\left(\mathrm{pO}_{2}{ }^{\text {in }}-\mathrm{pO}_{2}{ }^{\text {out }}\right) * \mathrm{~F} / \mathrm{W}$ where $\mathrm{pO}_{2}{ }^{\text {in }}$ and $\mathrm{pO}_{2}{ }^{\text {out }}$ were the oxygen contents

268 per $\mathrm{ml}$ of inflowing and outflowing perfusate, respectively, and the difference between them

269 multiplied by the perfusion rate $(\mathrm{F}, \mathrm{in} \mathrm{ml} / \mathrm{min})$ provided the total oxygen uptake per minute. This

270 value was then normalized by liver weight (W) to calculate the oxygen uptake per minute, per

271 gram of liver. After thawing and SNMP recovery, rat liver tissue was either flash frozen in liquid

272 nitrogen or fixed in 10\% formalin, embedded in paraffin, sectioned, and stained with

273 Hematoxylin and Eosin (H\&E). Terminal deoxynucleotidyl transferase dUTP nick end labeling

274 (TUNEL) was also performed on rat liver tissue after freezing to detect DNA breaks as an

275 indicator of apoptosis [9]. Liver tissue that was flash frozen was used to quantify ATP and EC by

276 the Massachusetts General Hospital (MGH) Mass Spectrometry Core (Boston, MA, USA). 
279 Tukey's post-hoc test (ANOVA/Tukey) was used for the comparison of the time-course

280 perfusion data. ATP and $\mathrm{EC}$ in the $-10^{\circ} \mathrm{C}$ vs. $-15^{\circ} \mathrm{C}$ group were compared using unpaired, two-

281 tailed t-tests.

\section{Results}

\section{A. Comparison of partially frozen rat livers at $-10^{\circ} \mathrm{C}$ vs $-15^{\circ} \mathrm{C}$ with}

\section{$12 \%$ glycerol}

Pooled results for livers stored for 1 and 5 days at $-10^{\circ} \mathrm{C}(\mathrm{n}=4)$ or $-15^{\circ} \mathrm{C}(\mathrm{n}=9)$ with a cocktail containing 12\% glycerol were compared 1 hour after CPA unloading and 3 hours after recovery with SNMP at $21^{\circ} \mathrm{C}$. There was no statistically significant difference between the two groups with respect to oxygen consumption (figure 2A), PV lactate (figure 2B), vascular resistance (figure 2C), and perfusate potassium on two-way ANOVA/Tukey testing. Similarly,

291 there were no statistically significant differences between $-10^{\circ} \mathrm{C}$ and $-15^{\circ} \mathrm{C}$ livers with respect to 292 percent weight gain (figure 2D), ATP (figure 2E), and EC (figure 2F) by unpaired, two-tailed t-

293 testing. Thus, rat livers partially frozen with $12 \%$ glycerol at $-10^{\circ} \mathrm{C}$ and $-15^{\circ} \mathrm{C}$ were not

294 functionally different, and as a result, were grouped together to form a more statistically robust

295 control group $(\mathrm{n}=13)$ for comparison against the ice modulator groups.

Fig 2. Perfusion metrics comparing partially frozen rat livers stored for $1(n=6)$ and

$2975(n=7)$ days at $-10^{\circ} \mathrm{C}(\mathrm{n}=4) \mathrm{vs}-15^{\circ} \mathrm{C}(\mathrm{n}=9)$ with $12 \%$ glycerol and 4 hours of recovery

298 during SNMP revealed no functional differences between the groups. (A) oxygen 
consumption, defined as outflow oxygen minus inflow oxygen, adjusted for liver weight and

300 flow rate, $(\mathrm{B})$ inflow lactate, $(\mathrm{C})$ resistance, defined as the perfusion pressure in the PV divided

301 by the flow rate and adjusted for liver weight after procurement, (D) percent weight gain, defined

302 as percentage increase of liver weight at the end of recovery compared to liver weight after

303 procurement, (E) ATP, (F) energy charge. Two-way ANOVA, followed by Tukey's post-hoc test

304 for A-C. Unpaired two-tailed t-test for D-F. Boxes: median with interquartile range. Whiskers:

$305 \min$ to max. Significance level: $\mathrm{p}<0.05$.

306

\section{B. Comparison of partially frozen control rat livers to livers frozen}

\section{with AFGP or X/Z-1000 ice modulators (pooled results for 1 and 5}

\section{days)}

There was no statistically significant difference between the three groups with respect to

312 only livers had the lowest mean perfusate lactate $(1.17 \pm 0.61 \mathrm{mmol} / \mathrm{L})$ at time zero. Over the

313 first hour of SNMP, there was a decrease in lactate among all three groups. There were no

314 significant differences between the groups at any time point according to the two-way

315 ANOVA/Tukey test (figure 3B). X/Z-1000 frozen livers had significantly higher mean vascular

316 resistance $(1.44 \pm 0.37 \mathrm{mmHg} /[(\mathrm{ml} / \mathrm{min}) / \mathrm{g}]$ at 0 hours compared to both $12 \%$ glycerol control

317 livers $(0.528 \pm 0.39 \mathrm{mmHg} / \mathrm{ml} / \mathrm{min}$, p-value 0.0315$)$ and AFGP livers $(0.413 \pm 0.27$, p-value

318 0.0215) (by ANOVA/Tukey). However, these differences were no longer significant at the

319 remaining time points as the resistance levels converged over time (figure 3C). At 1 hour,

320 glycerol only livers had higher mean perfusate potassium levels $(6.5 \pm 1.01 \mathrm{mmol} / \mathrm{L})$ compared 
321 to both AFGP livers $(5.1 \pm 0.22 \mathrm{mmol} / \mathrm{L}$, p-value 0.0167$)$ and $\mathrm{X} / \mathrm{Z}-1000$ livers $(5.6 \pm 0.14$

$322 \mathrm{mmol} / \mathrm{L}$, p-value 0.0275) (ANOVA/Tukey). However, these differences were no longer

323 significant at the remaining time points as the potassium levels converged over time.

324 Final mean weight gains for the glycerol-only control, the + AFGP, and the $+\mathrm{X} / \mathrm{Z}-1000$

325 groups were $26.9 \pm 15.3 \%, 12.8 \pm 13.2 \%$, and $39.5 \pm 5.69 \%$ respectively. Livers frozen with

326 AFGP had the least edema, significantly less compared to X/Z-1000 livers (p-value 0.0294 by

327 one-way ANOVA/Tukey; figure 3D). Mean ATP for the glycerol control group, AFGP, and

$328 \mathrm{X} / \mathrm{Z}-1000$ were $0.187 \pm 0.075,0.624 \pm 0.20$, and $0.680 \pm 0.32 \mathrm{ug} / \mathrm{ml}$ respectively. ATP

329 concentrations were significantly lower for livers stored with glycerol only compared with AFGP

$330(p=0.0057)$ and X/Z-1000 $(p=0.0023)($ ANOVA/Tukey). There was no significant difference

331 in ATP levels between AFGP and X/Z-1000 frozen livers $(\mathrm{p}=0.91)$ (figure 3E). Finally, mean

332 EC for the glycerol control group, AFGP, and X/Z-1000 was $0.066 \pm 0.032,0.063 \pm 0.015$, and

$3330.591 \pm 0.62(\mathrm{ATP}+1 / 2 \mathrm{ADP}) /(\mathrm{ATP}+\mathrm{ADP}+\mathrm{AMP})$ respectively. $\mathrm{EC}$ was dramatically higher in

334 livers stored with $\mathrm{X} / \mathrm{Z}-1000$ and glycerol compared to glycerol alone $(\mathrm{p}=0.0159)$ and to glycerol

335 plus AFGP $(p=0.0428)$ (one-way ANOVA/Tukey). There was no significant difference in EC

336 between AFGP and glycerol frozen livers $(\mathrm{p}=0.99)$ (figure 3F).

Fig 3. Perfusion metrics comparing rat livers partially frozen at $-15^{\circ} \mathrm{C}$ with $\mathrm{AFGP}$

$338(n=4)$ or with $X / Z-1000$ ice modulators $(n=4)$ versus $12 \%$ glycerol control $(n=13)$, with 4

339 hours of recovery during SNMP. There was higher $\mathrm{t}=0$ resistance and energy charge in X/Z-

3401000 livers, and lower ATP levels in glycerol livers. (A) oxygen consumption, defined as

341 outflow oxygen minus inflow oxygen, adjusted for liver weight and flow rate, (B) portal vein

342 lactate, $(\mathrm{C})$ vascular resistance, defined as the perfusion pressure in the PV divided by the flow

343 rate and adjusted for liver weight after procurement, (D) percent weight gain, defined as 
344 percentage increase of liver weight at the end of recovery compared to liver weight after

345 procurement, (E) ATP, (F) energy charge. Stars denote statistical significance (two-way ANOVA, followed by Tukey’s post-hoc test for A-C, one-way ANOVA, followed by Tukey’s

347 post-hoc test for D-F): $* 0.01<\mathrm{p}<0.05 ; * * 0.001<\mathrm{p}<0.01$. Error bars represent standard

348 deviation. Boxes: median with interquartile range. Whiskers: min to max.

H\&E staining of rat liver parenchyma following both 1 and 5 day partial freezing showed 350 sinusoidal, hepatocellular, and endothelial cell damage in all groups. In glycerol plus X/Z-1000

351 frozen livers, H\&E showed better preservation of sinusoidal patency (seemingly caused by less 352 hepatocyte cell swelling) than seen in the other groups after 1 day of storage, which deteriorated 353 somewhat after 5 days of storage. Endothelial patency also deteriorated between days 1 and 5, 354 with almost complete endothelial cell destruction around the PV vasculature (after 1 day: figure 355 4A; after 5 days, figure 4E). After 1 day of storage, glycogen staining was decreased in glycerol 356 plus X/Z-1000 livers, but not in the glycerol plus AFGP or glycerol-only groups. Glycerol plus 357 AFGP frozen livers exhibited cellular swelling at the expense of sinusoidal patency and suffered 358 from endothelial cell layer disruption that was mild after 1 day (figure 4B) and considerably 359 worse after 5 days (figure 4F) of storage. Livers frozen with only glycerol at $-10^{\circ} \mathrm{C}$ had 360 sinusoidal damage and compression, peri-portal endothelial destruction (figure 4D) and linear 361 cracks in the liver parenchyma (figure 4H). Decreasing the storage temperature of these control 362 glycerol livers to $-15^{\circ} \mathrm{C}$ did not alter the $\mathrm{H} \& \mathrm{E}$ pathology after either 1 (figure 4C) or 5 (figure 363 4G) days of frozen storage. 
TUNEL staining was observed in both the endothelium and the sinusoids after 1 and 5 days of frozen storage in the presence of X/Z-1000, but appeared to be less intense after 5 days of storage (figures 5A and 5E). AFGP frozen livers had primarily endothelial staining with mild sinusoidal staining after 1 day (figure 5B), but after 5 days, TUNEL staining had increased both around the vasculature and within the sinusoids (figure 5F). Compared to the other groups,

371 AFGP frozen livers at $-15^{\circ} \mathrm{C}$ and five days of partial freezing had the most TUNEL staining.

372 Glycerol only livers stored at $-10^{\circ} \mathrm{C}$ had remarkably less TUNEL staining compared to X/Z-

3731000 and AFGP livers with only mild sinusoidal staining after 1 day (figure 5D), and 5 days

374 (figure 5H) of storage. As in the H\&E results, decreasing the storage temperature of glycerol

375 only livers to $-15^{\circ} \mathrm{C}$ did not exacerbate TUNEL staining after 1 day (figure 5C) or 5 days

376 (figure 5G) of storage.

Fig 5. 40X Terminal deoxynucleotidyl transferase dUTP nick end labeling (TUNEL)

378 staining of rat liver parenchyma after partial freezing for 1 day (A-D) and 5 days (E-H)

with X/Z-1000, AFGP ice modulators versus $12 \%$ glycerol only.

\section{Discussion}

Extending the preservation time of donor organs has tremendous clinical application in

383 the field of transplantation. For liver transplantation, lengthening the allograft preservation time

384 from the current standard of SCS at $4^{\circ} \mathrm{C}$ will reduce the burden placed on the healthcare system

385 from high costs of unplanned surgeries, decrease organ rejection rates by incorporating more

386 HLA typing into clinical practice, and possibly even open avenues for global matching programs

$387[3,35]$. 

encountered either lethal $[14,15]$ or unacceptably damaging [16,37-39] amounts of extracellular ice, or the challenge of introducing the enormous concentrations of CPA needed to preclude such

391 damage $[16,40,41]$. So far, these difficult challenges have not been adequately overcome, and

392 therefore, other approaches should be investigated and may produce practical results more

393 rapidly. Our current approach primarily utilized cold but not cryogenic temperatures to extend

394 organ preservation time. We explored rat liver preservation at high subzero temperatures $\left(-10^{\circ} \mathrm{C}\right.$

395 and $-15^{\circ} \mathrm{C}$ ) combined with recovery SNMP to maximize the benefits of metabolic rate

396 depression and ex vivo organ assessment, while avoiding the dangers of deep cryogenic

397 temperatures. Ice modulators have been shown to modify ice crystal shape and inhibit ice

398 recrystallization, potentially decreasing ice-induced cellular damage. In the context of the partial

399 freezing of rat livers, ice modulators create an intriguing opportunity to preserve organs at

400 subzero temperatures in the presence of ice, allowing these stored organs to reap the benefits of

401 deeper metabolic stasis than current hypothermic standards while avoiding ice-related cellular

402 damage.

403 This study first demonstrated that rat livers frozen at $-10^{\circ} \mathrm{C}$ versus $-15^{\circ} \mathrm{C}$ with glycerol

404 were functionally similar regarding perfusion metrics, cellular architecture, and DNA damage,

405 indicating that the reduction in partial freezing did not significantly reduce metabolic function.

406 However, the reduction in storage temperature from $-10^{\circ} \mathrm{C}$ to $-15^{\circ} \mathrm{C}$ can have implications for

407 organ viability on a cellular level. As water freezes, solutes are excluded from the ice crystals,

408 which increases the osmolality and reduces the freezing point of the unfrozen water fraction.

409 Thus, a lower freezing temperature results in more ice and a higher level of osmotic shift [42]. In

410 our case, $12 \% \mathrm{v} / \mathrm{v}$ glycerol (1.64M), equates to 1.86 molality. According to the freezing point 
411 depression approximation, freezing point is lowered by about $1.86^{\circ} \mathrm{C}$ for every 1 osmolal

412 increase in concentration. Adding the 0.3 osmolal contribution of the glycerol vehicle solution,

413 the melting point of our storage media should be in the vicinity of $-4^{\circ} \mathrm{C}$. At $-10^{\circ} \mathrm{C}$, about $60 \%$ of

414 the water in the solution will be converted to ice, and at $-15^{\circ} \mathrm{C}$, about $73 \%$ of the water will be

415 frozen out, which is a significant increase. Although the membrane stabilizing saccharides,

416 trehalose and raffinose [43] were employed, they do not enter cells and therefore do not

417 nominally protect the inner membrane leaflet or reduce cell shrinkage induced by water

418 extraction during freezing.

419 A major aim of this research was to assess the effect of ice modulators such as AFGP and

$420 \mathrm{X} / \mathrm{Z}-1000$ on partially frozen rat livers compared to glycerol-only controls. AFGP frozen livers

421 had the least amount of edema and high levels of ATP. However, the AFGP-mediated ice

422 modulation had adverse effects on endothelial cells, which was reflected in both H\&E and

423 TUNEL staining, particularly after prolonged storage. As the duration of freezing increased from

4241 to 5 days, the AFGP TUNEL staining expanded from predominately endothelial damage to

425 diffuse sinusoidal cellular damage as well. AFGP has an established role in dynamic ice shaping,

426 ice recrystallization inhibition, and hysteretic freezing point depression $[22,44,45]$. Since

427 endothelial cells would make direct contact with the ice, it is possible that AFGP may be causing

428 less favorable ice crystal shapes that are disrupting the endothelial cells of the liver. In a study by

429 Rubinsky et al., antifreeze proteins resulted in the killing of all red blood cells during freezing

430 despite the use of directional solidification methods that normally minimize ice damage $[26,46]$.

431 Yet, AFGP may offer protection to hepatocytes through its other mechanism of action, ice

432 recrystallization inhibition. (AFGP freezing point depression is typically limited to $1-2^{\circ} \mathrm{C}$, which

433 is smaller than the difference between our solutions' $\mathrm{T}_{\mathrm{M}} \mathrm{S}$ and our chosen storage temperatures 
434 and therefore was not able to contribute a protective effect in these experiments). Isothermal

435 freeze fixation [15] could be useful in future studies for relating the details of ice distribution and

436 characteristics to observed outcomes [47].

$\mathrm{X} / \mathrm{Z}-1000$ was the second ice modulator combination assessed in this study. X/Z-1000

438 frozen livers had the highest ATP and by far the highest EC but suffered from the highest SNMP

439 resistance at $\mathrm{t}=0$ and had the most edema after recovery. On staining, $\mathrm{X} / \mathrm{Z}-1000$ frozen livers had

440 less TUNEL staining compared to AFGP frozen livers, but still exhibited both endothelial and

441 sinusoidal staining in excess of that seen for the glycerol only group. X/Z-1000 livers had a large

442 variation in both ATP and EC levels, which could potentially be explained by the competing

443 mechanisms of action of X-1000/Z-1000 with the potent ice nucleator, Snomax [30]. Snomax

444 would tend to reduce the number of ice crystals and, therefore, to increase their mean size and

445 the range of grain sizes. This might relate to the larger observed size of the sinusoids and to

446 stochastic differences in local nucleation and ice crystal size that affected the consistency of

447 hepatocyte viability. While X/Z-1000 is appealing for its high ATP and EC, the high level of

448 edema after partial freezing is concerning and might also be related to vascular damage caused

449 by larger local intravascular or interstitial ice grains, which would be consistent with previous

450 observations by Rubinsky et al. relating injury in frozen livers to vascular distension by

451 intravascular ice [48]. A future direction related to this ice modulator should be to explore the

452 use of $\mathrm{X}-1000$, which is a recrystallization inhibitor, without the use of Z-1000, which is an

453 antinucleator. On the other hand, total vascular distension should have been similar in all groups,

454 as dictated by the phase diagram of glycerol-water solutions, and yet edema was more moderate

455 in the glycerol-only group. In any case, X/Z-1000 seems promising for use with the isolation or 
456 preservation of isolated hepatocytes, for which maintenance of high ATP/EC would be the main

457 goal.

458 Finally, glycerol-only control livers had the lowest lactate levels at $\mathrm{t}=0$ and minimal

459 TUNEL staining. However, these livers also had very low ATP and EC compared to the ice

460 modulator groups. A potential biological reason for this difference is that glycerol induces

461 glycerol kinase to convert glycerol to glycerol-3-phospahte, which is an ATP dependent

462 pathway. Thus, the activity of glycerol as a CPA could depress ATP levels [49]. The

463 extraordinary and unexpected ability of both ice modulators to prevent this has no clear

464 explanation, but it seemed that utilization of glycogen to generate ATP and lactate was more

465 effective in the X/Z-1000 group and to a lesser extent in the AFGP group, based on more intense

466 glycogen staining (suggesting less glycogen metabolism) in the glycerol-only group. It would be

467 interesting to see if structurally unrelated small molecule ice recrystallization inhibitors (IRIs)

468 [50] would have a similar effect and also better protect the vascular system. Without the effects

469 of the ice modulators preventing damaging ice recrystallization, glycerol-only livers also

470 consistently had parenchymal cracks and endothelial cell obliteration on H\&E staining, despite

471 adding Snomax and 3-OMG to rescue endothelial cells from partial freezing injury. Thus, the

472 concept of adding ice modulation to the basic methodology for high temperature freezing

473 appears to be well supported. Finally, all liver groups cleared lactate over the 2 hour perfusion

474 and (while incurring hepatocellular damage) had viable H\&E histology after perfusion, meeting

475 two criteria for transplantation $[51,52]$. Thus, future experiments transplanting these partially

476 frozen livers after SNMP can be conducted to assess if perfusion performance corelates with in

477 vivo hepatic function. 
Overall, the ideal ice modulator combination to enhance the partial freezing protocol

479 would retain the positive effects of high ATP and high EC seen in X/Z-1000, the low levels of

480 edema with AFGP, without the cellular damage to endothelial and sinusoidal cells seen with both

481 ice modulator groups. Thus, future directions to expand the preservation of livers for

482 transplantation with the partial freezing approach depend on both modifications to the freezing

483 protocol as well as the ice modulator combination. Specifically, increasing the CPA

484 concentration to decrease ice formation at lower temperatures and improving the loading and

485 unloading of CPAs with SNMP could improve liver viability. Additionally, there are other

486 permeating CPAs that could be tested in the partial freezing protocol such as dimethyl sulfoxide,

487 ethylene glycol, N-methylformamide, propylene glycol, and urea [40,53,54]. Finally, altering the

488 base solution from UW to a lower potassium carrying solution could decrease the

489 transmembrane osmotic stress in the unfrozen water fraction.

490 In summary, this research incorporated ice modulators into the rat liver partial freezing

491 protocol to prolong the preservation time of livers. We demonstrated that there was no difference

492 in partially frozen livers with only glycerol at $-10^{\circ} \mathrm{C}$ versus $-15^{\circ} \mathrm{C}$ with respect to perfusion

493 metrics, cellular architecture, and DNA damage. Additionally, we showed that AFGP and X/Z-

4941000 ice modulators can have beneficial effects on partially frozen rat liver ATP and EC levels,

495 respectively. However, further work on elucidating the optimal ice modulator cocktail is

496 necessary as AFGP livers had high levels of endothelial DNA damage and X/Z-1000 livers

497 suffered from post-freeze edema. Modifications to CPA combinations, as well as improvements

498 to machine perfusion CPA loading and unloading, can help improve the viability of these

499 partially frozen organs. 


\section{Acknowledgements}

503 R01DK096075, R01DK107875) and NSF ATP-Bio ERC grant (NSF 1941543). Further, we

504 gratefully acknowledge funding to SNT for Career Development from NIH (K99HL1431149-

505 01A1), American Heart Association (18CDA34110049), Harvard Medical School Eleanor and

506 Miles Shore Fellowship, and the Claflin Distinguished Scholar Award on behalf of the MGH

507 Executive Committee on Research. We gratefully acknowledge research support to Omar Haque

508 by the American Liver Foundation (2019 Hans Popper Memorial Postdoctoral Research

509 Fellowship) and the American College of Surgeons (Grant number 1123-39991 scholarship

510 endowment fund).

511

\section{Data availability}

513 The authors declare that the data supporting the findings of this study are available within

514 the paper and its supplementary information files. Any additional data, if needed, will be

515 provided upon request.

516 


\section{References}

526

527

528

529

530

531

532

533

534

535

536

537

538

539

540

541

542

543

544

545

546

547

548

549

550

551

552

553

554

555

556

557

558

559

560

561

562

563

564

565

566

567

568

569

1. Ray K. Preserving the liver for transplantation. Nature Reviews Gastroenterology and Hepatology. 2018;15(327). doi:10.1038/s41575-018-0024-7

2. Taylor MJ, Weegman BP, Baicu SC, Giwa SE. New Approaches to Cryopreservation of Cells, Tissues, and Organs. Transfusion Medicine and Hemotherapy. 2019;46(3). doi:10.1159/000499453

3. Giwa S, Lewis JK, Alvarez L, Langer R, Roth AE, Church GM, et al. The promise of organ and tissue preservation to transform medicine. Nature Biotechnology. 2017;35:530542. doi:10.1038/nbt.3889

4. Yamada K, Sykes M, Sachs DH. Tolerance in xenotransplantation. Current Opinion in Organ Transplantation. 2017;22(6):522-528. doi:10.1097/MOT.0000000000000466

5. Ardehali A, Esmailian F, Deng M, Soltesz E, Hsich E, Naka Y, et al. Ex-vivo perfusion of donor hearts for human heart transplantation (PROCEED II): A prospective, open-label, multicentre, randomised non-inferiority trial. Lancet. 2015;27(9987):2577-2584.

doi:10.1016/S0140-6736(15)60261-6

6. Warnecke G, Van Raemdonck D, Smith MA, Massard G, Kukreja J, Rea F, et al. Normothermic ex-vivo preservation with the portable Organ Care System Lung device for bilateral lung transplantation (INSPIRE): a randomised, open-label, non-inferiority, phase 3 study. Lancet Respir Med. 2018;6(5):357-367. doi:10.1016/S2213-2600(18)30136-X

7. Nasralla D, Coussios CC, Mergental H, Akhtar MZ, Butler AJ, Ceresa CDL, et al. A randomized trial of normothermic preservation in liver transplantation. Nature.

2018;557(7703):50-56. doi:10.1038/s41586-018-0047-9

8. Eshmuminov D, Becker D, Bautista Borrego L, Hefti M, Schuler MJ, Hagedorn C, et al. An integrated perfusion machine preserves injured human livers for 1 week. Nat Biotechnol. 2020;38:189-198. doi:10.1038/s41587-019-0374-x

9. de Vries RJ, Tessier SN, Banik PD, Nagpal S, Cronin SEJ, Ozer S, et al. Supercooling extends preservation time of human livers. Nat Biotechnol. 2019;37:1131-1136. doi:10.1038/s41587-019-0223-y

10. de Vries R, Tessier SN, Banik PD, Ozer S, Crorin SEJ, Nagpal S, et al. Extending the Human Liver Preservation Time for Transplantation by Supercooling. Transplantation. 2018;102:615. doi:10.1097/01.tp.0000543168.17458.55

11. Berendsen TA, Bruinsma BG, Puts CF, Saeidi N, Usta OB, Uygun BE, et al. Supercooling enables long-term transplantation survival following 4 days of liver preservation. Nat Med. 2014;20(7):790-793. doi:10.1038/nm.3588

12. Bruinsma BG, Berendsen TA, Izamis ML, Yeh H, Yarmush ML, Uygun K. Supercooling preservation and transplantation of the rat liver. Nat Protoc. 2015;10(3):484-494. doi:10.1038/nprot.2015.011

13. Zachariassen KE, Kristiansen E. Ice nucleation and antinucleation in nature. Cryobiology. 2000;41(4):257-279. doi:10.1006/cryo.2000.2289

14. Pegg DE. The relevance of ice crystal formation for the cryopreservation of tissues and organs. Cryobiology. 2010;60(3 supp):S36-44. doi:10.1016/j.cryobiol.2010.02.003

15. Hunt CJ. Studies on cellular structure and ice location in frozen organs and tissues: The use of freeze-substitution and related techniques. Cryobiology. 1984;21(4):385-402. doi:10.1016/0011-2240(84)90077-4 
570 16. Fahy GM, Wowk B, Pagotan R, Chang A, Phan J, Thomson B, et al. Physical and biological aspects of renal vitrification. Organogenesis. 2009;5(3):167-175. doi:10.4161/org.5.3.9974

17. Fahy GM. Analysis of "solution effects" injury: Cooling rate dependence of the functional and morphological sequellae of freezing in rabbit renal cortex protected with dimethyl sulfoxide. Cryobiology. 1981;18(6):550-570. doi:10.1016/0011-2240(81)90123-1

18. Tessier SN, Weng L, Moyo WD, Au SH, Wong KHK, Angpraseuth C, et al. Effect of Ice Nucleation and Cryoprotectants during High Subzero-Preservation in Endothelialized Microchannels. ACS Biomater Sci Eng. 2018;4(8):3006-3015. doi:10.1021/acsbiomaterials.8b00648

19. Ishine N, Rubinsky B, Lee CY. Transplantation of Mammalian Livers Following Freezing: Vascular Damage and Functional Recovery. Cryobiology. 2000;40(1):84-89. doi:10.1006/cryo.1999.2225

20. Celik Y, Drori R, Pertaya-Braun N, Altan A, Barton T, Bar-Dolev M, et al. Microfluidic experiments reveal that antifreeze proteins bound to ice crystals suffice to prevent their growth. Proc Natl Acad Sci U S A. 2013;110(4):1309-1314.

doi:10.1073/pnas.1213603110

21. Kuiper MJ, Morton CJ, Abraham SE, Gray-Weale A. The biological function of an insect antifreeze protein simulated by molecular dynamics. Elife. 2015;4: e05142.

doi:10.7554/eLife. 05142

22. Weng L, Stott SL, Toner M. Molecular Dynamics at the Interface between Ice and Poly(vinyl alcohol) and Ice Recrystallization Inhibition. Langmuir. 2018;34(17): 51165123. doi:10.1021/acs.langmuir.7b03243

23. Naullage PM, Lupi L, Molinero V. Molecular Recognition of Ice by Fully Flexible Molecules. J Phys Chem C. 2017;121(48):26949-26957. doi:10.1021/acs.jpcc.7b10265

24. Weng L, Swei A, Toner M. Role of synthetic antifreeze agents in catalyzing ice nucleation. Cryobiology. 2018;84:91-94. doi:10.1016/j.cryobiol.2018.08.010

25. Eickhoff L, Dreischmeier K, Zipori A, Sirotinskaya V, Adar C, Reicher N, et al. Contrasting Behavior of Antifreeze Proteins: Ice Growth Inhibitors and Ice Nucleation Promoters. J Phys Chem Lett. 2019;10(5):966-972. doi:10.1021/acs.jpclett.8b03719

26. Ishiguro H, Rubinsky B. Mechanical interactions between ice crystals and red blood cells during directional solidification. Cryobiology. 1994;31(5):483-500.

doi:10.1006/cryo.1994.1059

27. Wowk B, Leitl E, Rasch CM, Mesbah-Karimi N, Harris SB, Fahy GM. Vitrification enhancement by synthetic ice blocking agents. Cryobiology. 2000;40(3):228-236. doi:10.1006/cryo.2000.2243

28. Burkey AA, Riley CL, Wang LK, Hatridge TA, Lynd NA. Understanding Poly(vinyl alcohol)-Mediated Ice Recrystallization Inhibition through Ice Adsorption Measurement and pH Effects. Biomacromolecules. 2018;19(1):248-55. doi:10.1021/acs.biomac.7b01502

29. Hasan M, Fayter AER, Gibson MI. Ice Recrystallization Inhibiting Polymers Enable Glycerol-Free Cryopreservation of Microorganisms. Biomacromolecules. 2018;19(8): 3371-3376. doi:10.1021/acs.biomac.8b00660

30. Wowk B, Fahy GM. Inhibition of bacterial ice nucleation by polyglycerol polymers. Cryobiology. 2002;44(1):14-23. doi:10.1016/S0011-2240(02)00008-1

31. Amir G, Horowitz L, Rubinsky B, Yousif BS, Lavee J, Smolinsky AK. Subzero 
nonfreezing cryopresevation of rat hearts using antifreeze protein I and antifreeze protein III. Cryobiology. 2004;48(3):273-282. doi:10.1016/j.cryobiol.2004.02.009

32. Amir G, Rubinsky B, Horowitz L, Miller L, Leor J, Kassif Y, et al. Prolonged 24-hour subzero preservation of heterotopically transplanted rat hearts using antifreeze proteins derived from arctic fish. Ann Thorac Surg. 2004;77(5):1648-1655. doi:10.1016/j.athoracsur.2003.04.004

33. Amir G, Rubinsky B, Basheer SY, Horowitz L, Jonathan L, Feinberg MS, et al. Improved viability and reduced apoptosis in sub-zero 21-hour preservation of transplanted rat hearts using anti-freeze proteins. J Hear Lung Transplant. 2005;24(11):1915-1929. doi:10.1016/j.healun.2004.11.003

34. Watson CJE, Jochmans I. From "Gut Feeling" to Objectivity: Machine Preservation of the Liver as a Tool to Assess Organ Viability. Curr Transplant Reports. 2018;5(1):72-81. doi:10.1007/s40472-018-0178-9

35. de Vries RJ, Yarmush M, Uygun K. Systems engineering the organ preservation process for transplantation. Current Opinion in Biotechnology. 2019;58:192-201. doi:10.1016/j.copbio.2019.05.015

36. Campbell BK, Hernandez-Medrano J, Onions V, Pincott-Allen C, Aljaser F, Fisher J, et al. Restoration of ovarian function and natural fertility following the cryopreservation and autotransplantation of whole adult sheep ovaries. Hum Reprod. 2014;29(8):1749-1763. doi:10.1093/humrep/deu144

37. Hamilton R, Holst HI, Lehr HB. Successful preservation of canine small intestine by freezing. J Surg Res. 1973;14(4):313-318. doi:10.1016/0022-4804(73)90033-4

38. Lin JH, Carroll PM. The effect of calcium and magnesium on frozen rat uteri, and the calcium content of uteri frozen by various procedures. Cryobiology. 1968;5(2):105-108. doi:10.1016/S0011-2240(68)80151-8

39. Taylor MJ, Pegg DE. The effect of ice formation on the function of smooth muscle tissue stored at -21 or $-60{ }^{\circ} \mathrm{C}$. Cryobiology. 1983;20(1):36-40. doi:10.1016/00112240(83)90057-3

40. Fahy GM, Wowk B, Wu J, Phan J, Rasch C, Chang A, et al. Cryopreservation of organs by vitrification: Perspectives and recent advances. Cryobiology. 2004;48(2):157-178. doi:10.1016/j.cryobiol.2004.02.002

41. Farrant J. Mechanism of cell damage during freezing and thawing and its prevention. Nature. 1965;205:1284-1287. doi:10.1038/2051284a0

42. Libbrecht KG. Physical Dynamics of Ice Crystal Growth. Annu Rev Mater Res. 2017;47:271-295. doi:10.1146/annurev-matsci-070616-124135

43. Van den Ende W. Multifunctional fructans and raffinose family oligosaccharides. Frontiers in Plant Science. 2013;4:247. doi:10.3389/fpls.2013.00247

44. Urbańczyk M, Góra J, Latajka R, Sewald N. Antifreeze glycopeptides: from structure and activity studies to current approaches in chemical synthesis. Amino Acids. 2017;49(2):209-222. doi:10.1007/s00726-016-2368-z

45. Bang JK, Lee JH, Murugan RN, Lee SG, Do H, Koh HY, et al. Antifreeze peptides and glycopeptides, and their derivatives: Potential uses in biotechnology. Marine Drugs. 2013;11(6):2013-2041. doi:10.3390/md11062013

46. Mugnano JA, Wang T, Layne JR, DeVries AL, Lee RE. Antifreeze glycoproteins promote intracellular freezing of rat cardiomyocytes at high subzero temperatures. Am J Physiol Regul Integr Comp Physiol. 1995;269(2):474-479. doi:10.1152/ajpregu.1995.269.2.r474 
662 47. Hunt CJ, Taylor MJ, Pegg DE. Freeze-substitution and isothermal freeze-fixation studies to elucidate the pattern of ice formation in smooth muscle at $252 \mathrm{~K}\left(-21^{\circ} \mathrm{C}\right)$. J Microsc. 1982;125(2):177-186. doi:10.1111/j.1365-2818.1982.tb00335.x

48. Rubinsky B, Lee CY, Bastacky J, Onik G. The process of freezing and the mechanism of damage during hepatic cryosurgery. Cryobiology. 1990;27(1):85-97. doi:10.1016/00112240(90)90055-9

49. Robinson J, Newsholme EA. Some properties of hepatic glycerol kinase and their relation to the control of glycerol utilization. Biochem J. 1969;112(4):455-464.

671

50. Briard JG, Poisson JS, Turner TR, Capicciotti CJ, Acker JP, Ben RN. Small molecule ice recrystallization inhibitors mitigate red blood cell lysis during freezing, transient warming and thawing. Sci Rep. 2016;29(6):23619. doi:10.1038/srep23619 for normothermic preservation of the rat liver. Tissue Eng. 2007;13(8):2143-2151. doi:10.1089/ten.2007.0101

52. Nösser M, Gassner JMGV, Moosburner S, Wyrwal D, Claussen F, Hillebrandt KH, et al. Development of a Rat Liver Machine Perfusion System for Normothermic and Subnormothermic Conditions. Tissue Eng Part A. 2020;26(1):57-65.

53. Katenz E, Vondran FWR, Schwartlander R, Pless G, Gong X, Cheng X, et al. Cryopreservation of primary human hepatocytes: The benefit of trehalose as an additional cryoprotective agent. Liver Transplant. 2007;13(1):38-45. doi:10.1002/1t.20921

54. Spindler R, Wolkers WF, Glasmacher B. Dimethyl sulfoxide and ethylene glycol promote membrane phase change during cryopreservation. Cryo-Letters. 2011;32(2):148-157. 
Supplemental Table 1: Composition of all solutions used in each phase of the partial freezing protocol. $\mathrm{WE}=$ Williams $\mathrm{E}, \mathrm{UW}=$ University of Wisconsin, $\mathrm{PEG}=$ polyethylene glycol, $\mathrm{BSA}=$ bovine serum albumin, $\mathrm{HES}=$ hydroxyethyl starch, 3-OMG = 3-O-methyl-D-glucose, $\mathrm{CPA}=$

Subnormothermic
Preconditioning
solution

Base solution

Total volume

Additives

Insulin

Heparin

Dexamethasone

Hydrocortisone

Penicillin

Streptomycin

Glutathione
WE

$250 \mathrm{ml}$
Hypothermic Preloading solution

WE

$250 \mathrm{ml}$
Storage Solution

UW

$100 \mathrm{ml}$
Subnormothermic

Thawing Recovery
Solution

WE

$250 \mathrm{ml}$
Solution

WE

$500 \mathrm{ml}$

\section{Macromolecules}

\begin{tabular}{llllll}
\hline $35 \mathrm{kDA}$ PEG & $20 \mathrm{~g} / \mathrm{l}$ & $20 \mathrm{~g} / 1$ & $50 \mathrm{~g} / \mathrm{l}$ & $20 \mathrm{~g} / 1$ & $20 \mathrm{~g} / \mathrm{l}$ \\
$\mathrm{BSA}$ & $50 \mathrm{~g} / 1$ & $50 \mathrm{~g} / 1$ & --- & $50 \mathrm{~g} / 1$ & $50 \mathrm{~g} / 1$ \\
HES & --- & $30 \mathrm{~g} / 1$ & $50 \mathrm{~g} / \mathrm{l}^{*}$ & $30 \mathrm{~g} / 1$ & ---
\end{tabular}

\section{Saccharides}

\begin{tabular}{llllll}
\hline 3-OMG & $19.42 \mathrm{~g} / \mathrm{l}$ & $19.42 \mathrm{~g} / \mathrm{l}$ & --- & $19.42 \mathrm{~g} / \mathrm{l}$ & --- \\
Raffinose & --- & $15.12 \mathrm{~g} / 1$ & $17.83 \mathrm{~g} / \mathrm{I}^{*}$ & $15.12 \mathrm{~g} / 1$ & --- \\
Trehalose & --- & --- & $18.92 \mathrm{~g} / 1$ & $18.92 \mathrm{~g} / 1$ & ---
\end{tabular}

CPA / INA

\begin{tabular}{llllll}
\hline Glycerol & --- & $60 \mathrm{ml} / 1$ & $120 \mathrm{ml} / 1$ & --- & -- \\
Snomax & --- & --- & $1 \mathrm{~g} / \mathrm{l}$ & --- & --
\end{tabular}




\section{Supplemental Table 2: List of suppliers for each reagent used in partial freezing solutions.}

\section{Reagent}

University of Wisconsin (UW) solution

Williams' Medium E

Insulin (Humulin R)

Sodium heparin

Dexamethasone

Hydrocortisone

Penicillin-Streptomycin

L-Glutathione

Bovine Serum Albumin (BSA)

35kDa Polyethylene glycol (PEG)

3-O-methyl glucose

D-(+)-Trehalose dihydrate

Glycerol

Antifreeze glycopeptides (AFGP)

$\mathrm{X} / \mathrm{Z}-1000$

Snomax

\section{Supplier}

Bridge to Life

Sigma-Aldrich

MGH pharmacy

MGH pharmacy

Sigma-Aldrich

MGH pharmacy

Invitrogen

Sigma-Aldrich

Sigma-Aldrich

Sigma-Aldrich

Chem-Impex

Sigma-Aldrich

Fisher Scientific

A/F Protein Inc.

21st Century Medicine

Telemet 


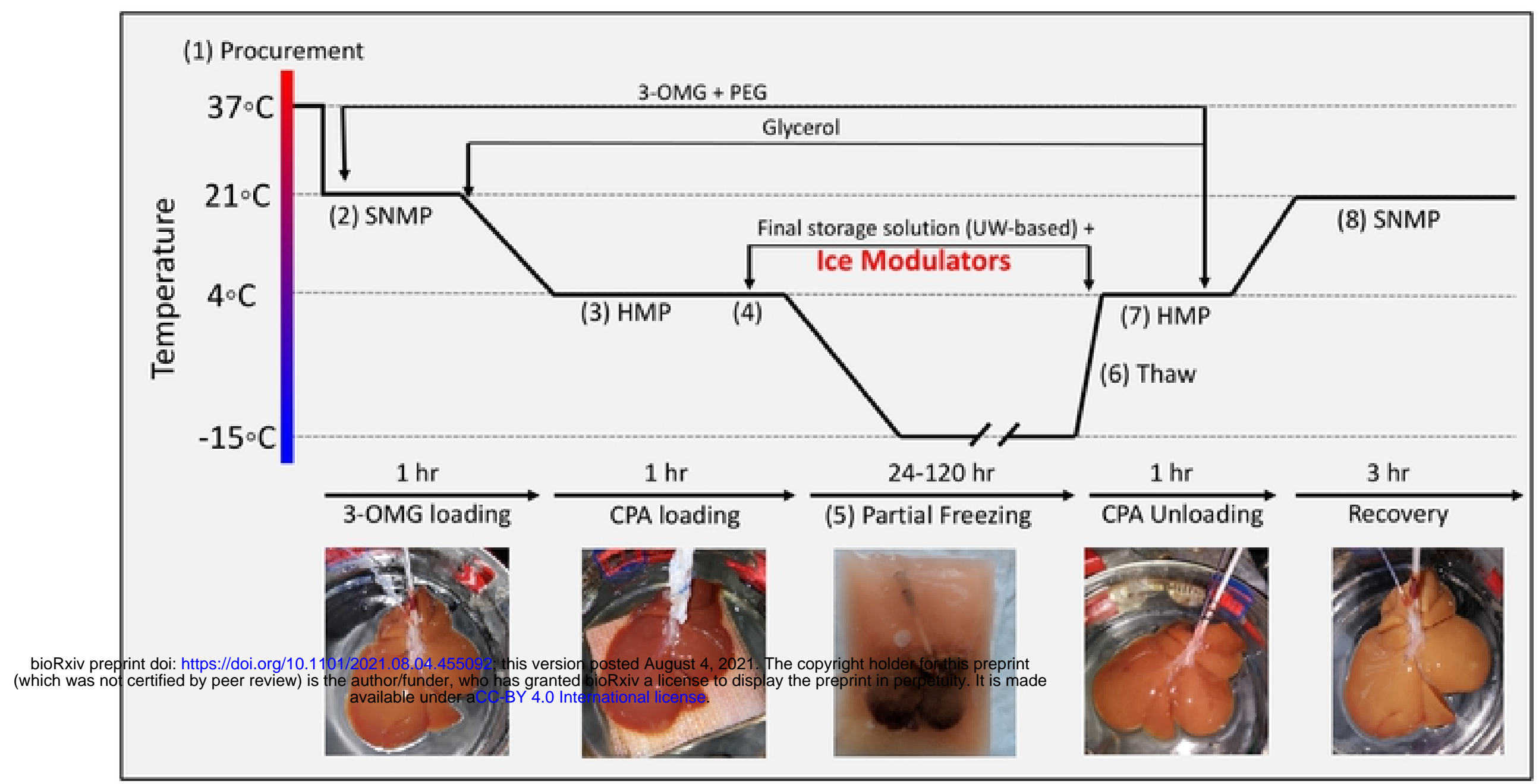

Figure 1: Experimental design of partial freezing, in 8 consecutive steps: (1) liver procurement, (2) preconditioning during SNMP, (3) CPA preloading during hypothermic machine perfusion (HMP), (4) loading of final storage solution and ice modulators during HMP, (5) partial freezing of rat liver, (6) thawing of rat liver, (7) unloading of CPAs during HMP, and (8) functional recovery of frozen rat livers during SNMP. 
A

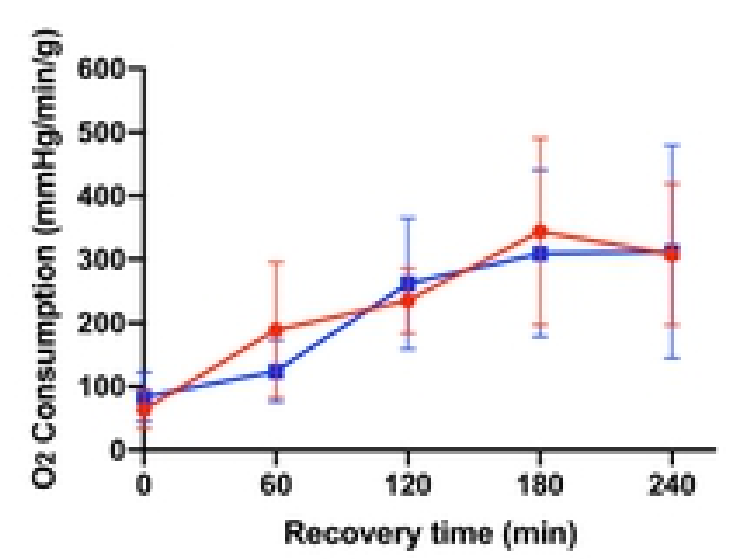

D

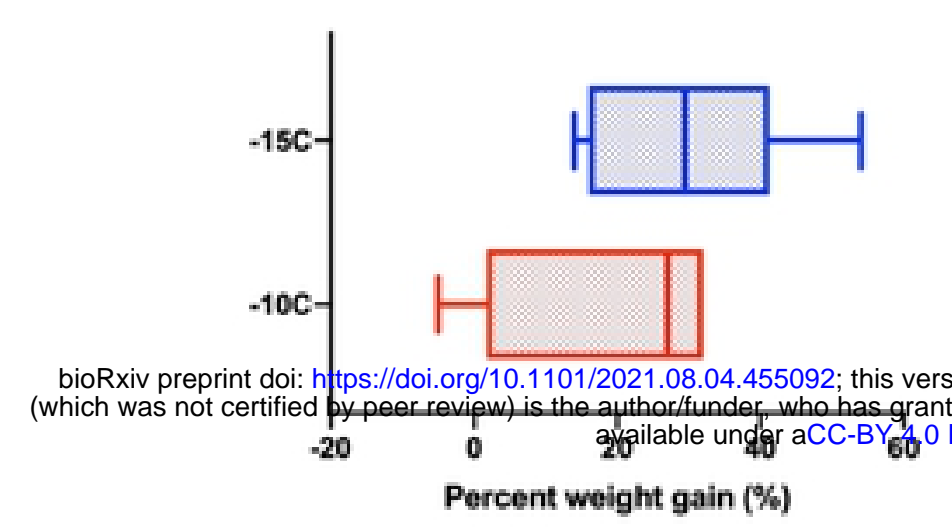

B

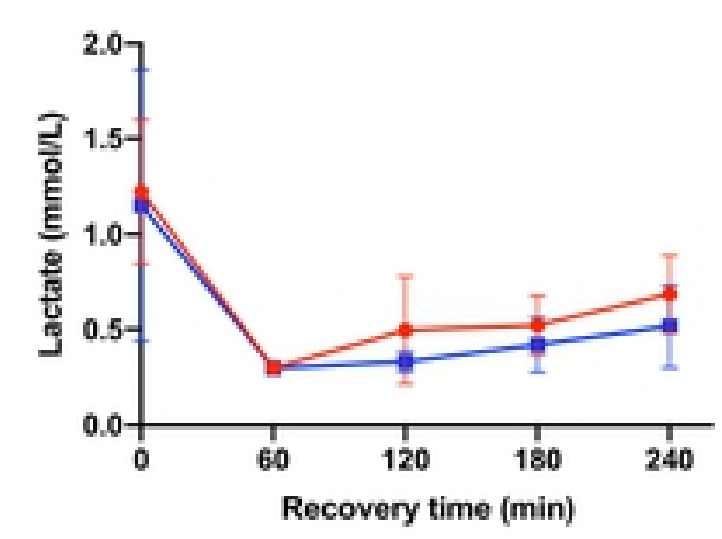

E

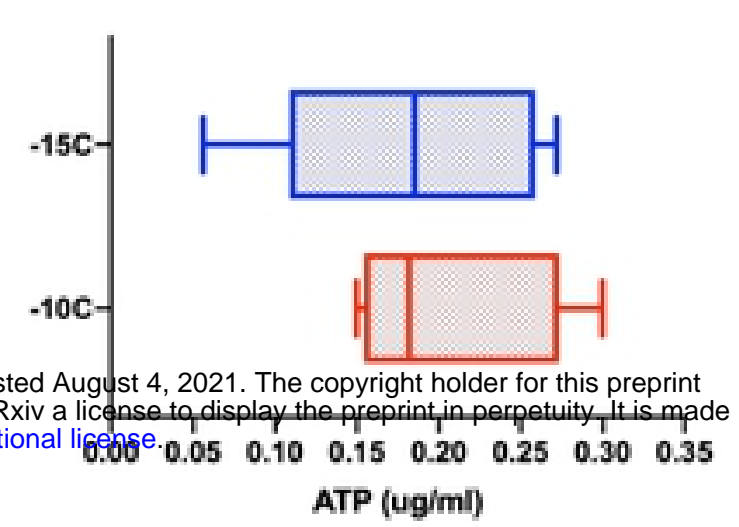

C

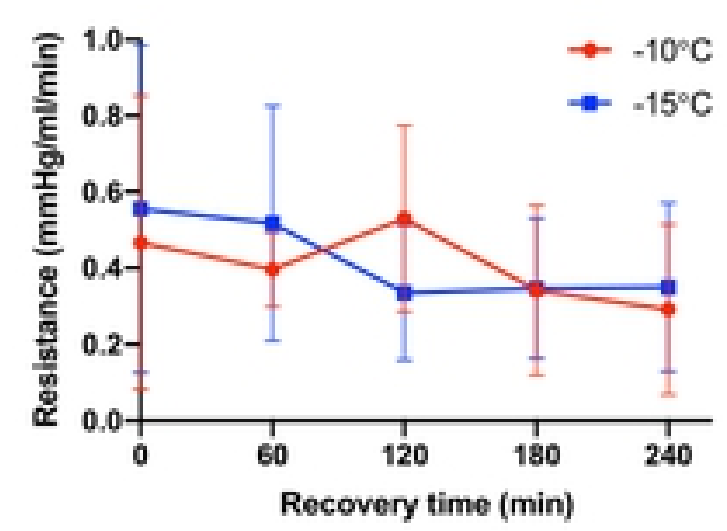

$\mathbf{F}$

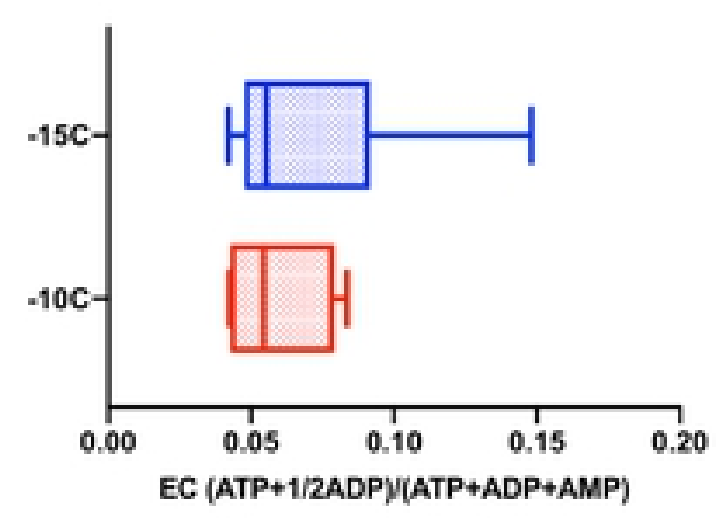

Figure 2: Perfusion metrics comparing partially frozen rat livers stored for $1(n=6)$ and $5(n=7)$ days at $-10^{\circ} \mathrm{C}(\mathrm{n}=4) \mathrm{vs}-15^{\circ} \mathrm{C}(\mathrm{n}=9)$ with $12 \%$ glycerol and 4 hours of recovery during SNMP revealed no functional differences between the groups. (A) oxygen consumption, defined as outflow oxygen minus inflow oxygen, adjusted for liver weight and flow rate, (B) inflow lactate, (C) resistance, defined as the perfusion pressure in the PV divided by the flow rate and adjusted for liver weight after procurement, (D) percent weight gain, defined as percentage increase of liver weight at the end of recovery compared to liver weight after procurement, (E) ATP, (F) energy charge. Two-way ANOVA, followed by Tukey's post-hoc test for A-C. Unpaired twotailed t-test for D-F. Boxes: median with interquartile range. Whiskers: min to max. Significance level: $\mathrm{p}<0.05$. 
A

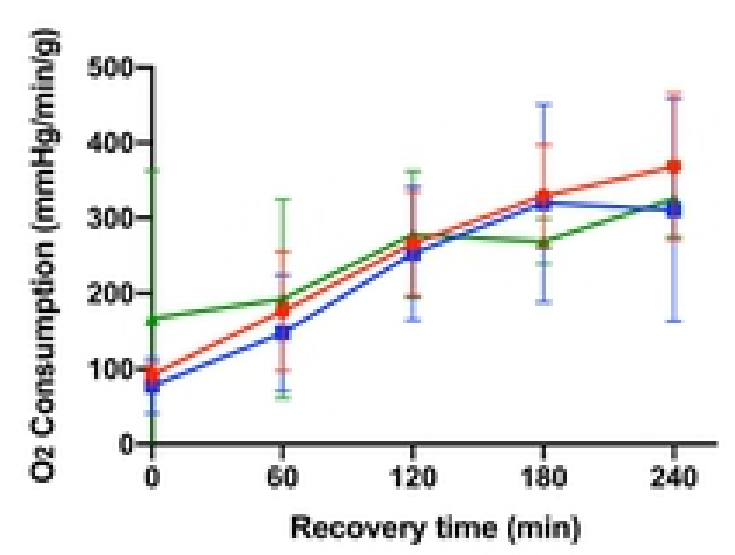

D

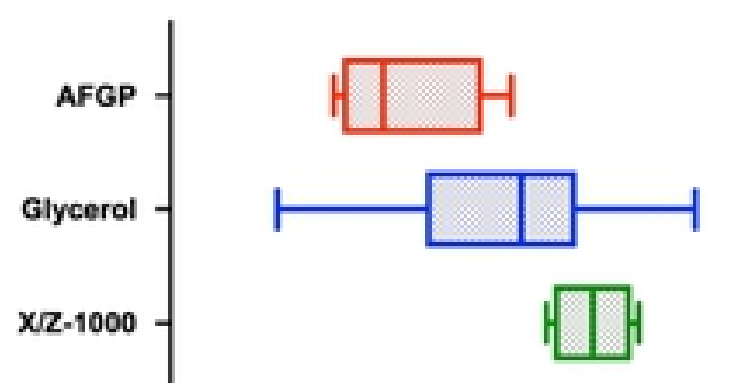

B

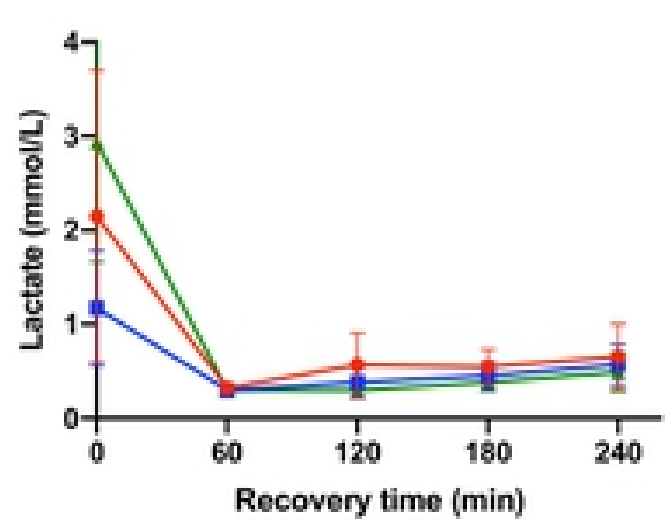

E

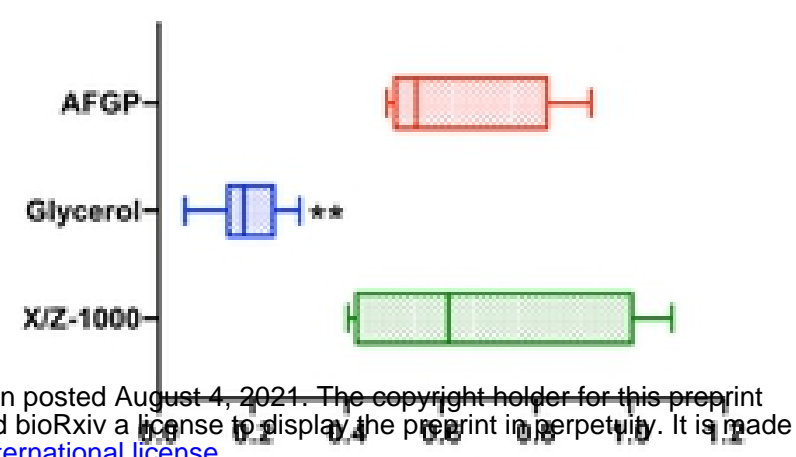

ATP (ug/ml)
C

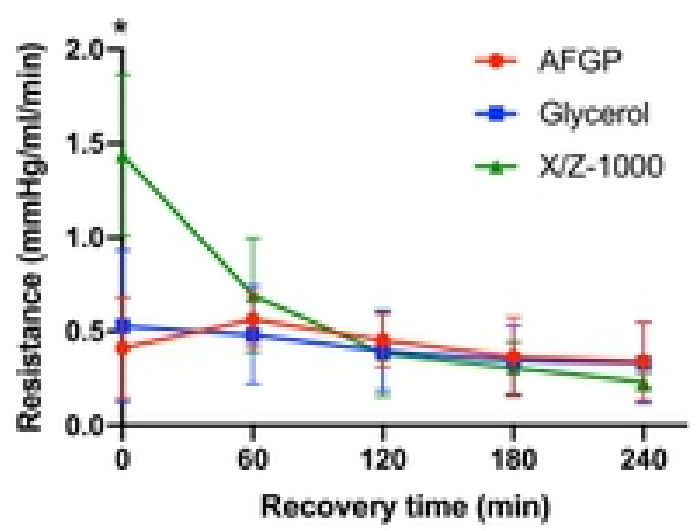

$\mathbf{F}$

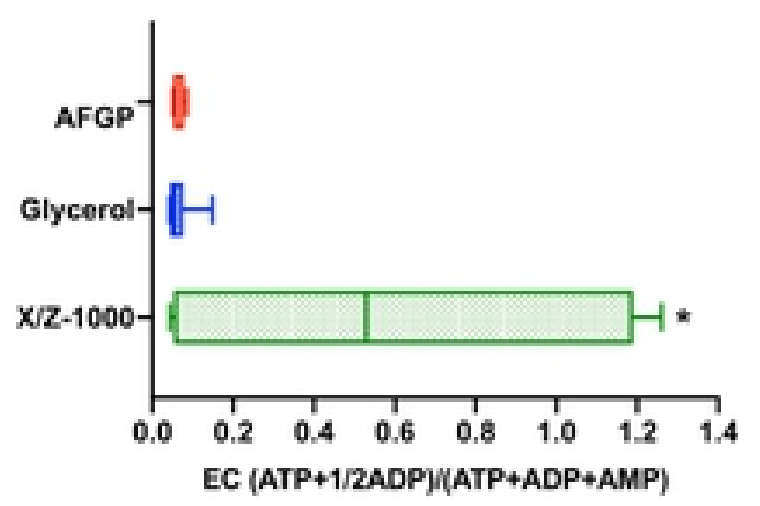

Figure 3: Perfusion metrics comparing rat livers partially frozen at $-15^{\circ} \mathrm{C}$ with $\mathrm{AFGP}(\mathrm{n}=4)$ or with X/Z-1000 ice modulators $(n=4)$ versus $12 \%$ glycerol control $(n=13)$, with 4 hours of recovery during SNMP. There was higher $\mathrm{t}=0$ resistance and energy charge in $\mathrm{X} / \mathrm{Z}-1000$ livers, and lower ATP levels in glycerol livers. (A) oxygen consumption, defined as outflow oxygen minus inflow oxygen, adjusted for liver weight and flow rate, (B) portal vein lactate, $(\mathbf{C})$ vascular resistance, defined as the perfusion pressure in the PV divided by the flow rate and adjusted for liver weight after procurement, (D) percent weight gain, defined as percentage increase of liver weight at the end of recovery compared to liver weight after procurement, (E) ATP, (F) energy charge. Stars denote statistical significance (two-way ANOVA, followed by Tukey's post-hoc test for A-C, one-way ANOVA, followed by Tukey's post-hoc test for D-F): $* 0.01<\mathrm{p}<0.05 ; * * 0.001<\mathrm{p}<0.01$. Error bars represent standard deviation. Boxes: median with interquartile range. Whiskers: $\min$ to $\max$. 


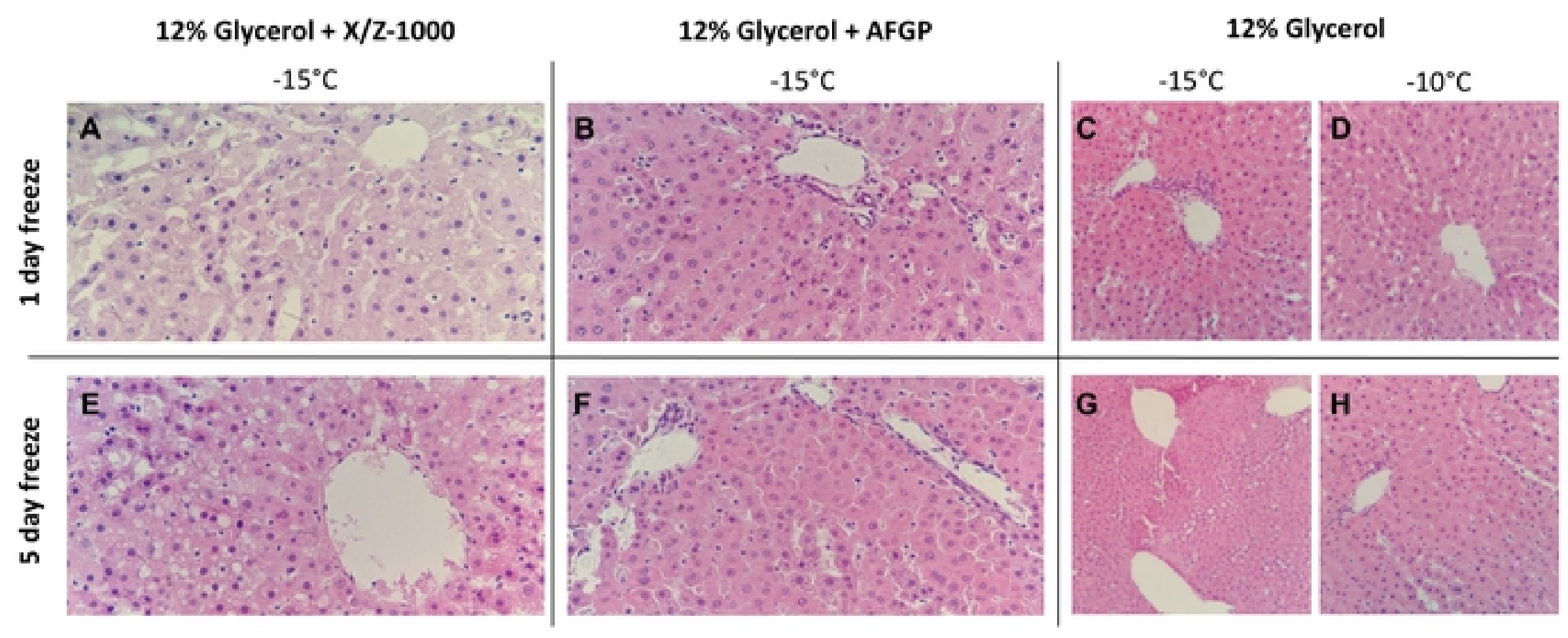

Figure 4: 40X H\&E staining of rat liver parenchyma after partial freezing for 1 day (A-D) and 5

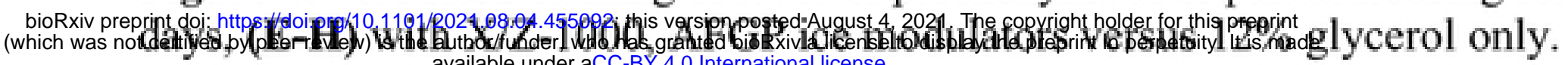




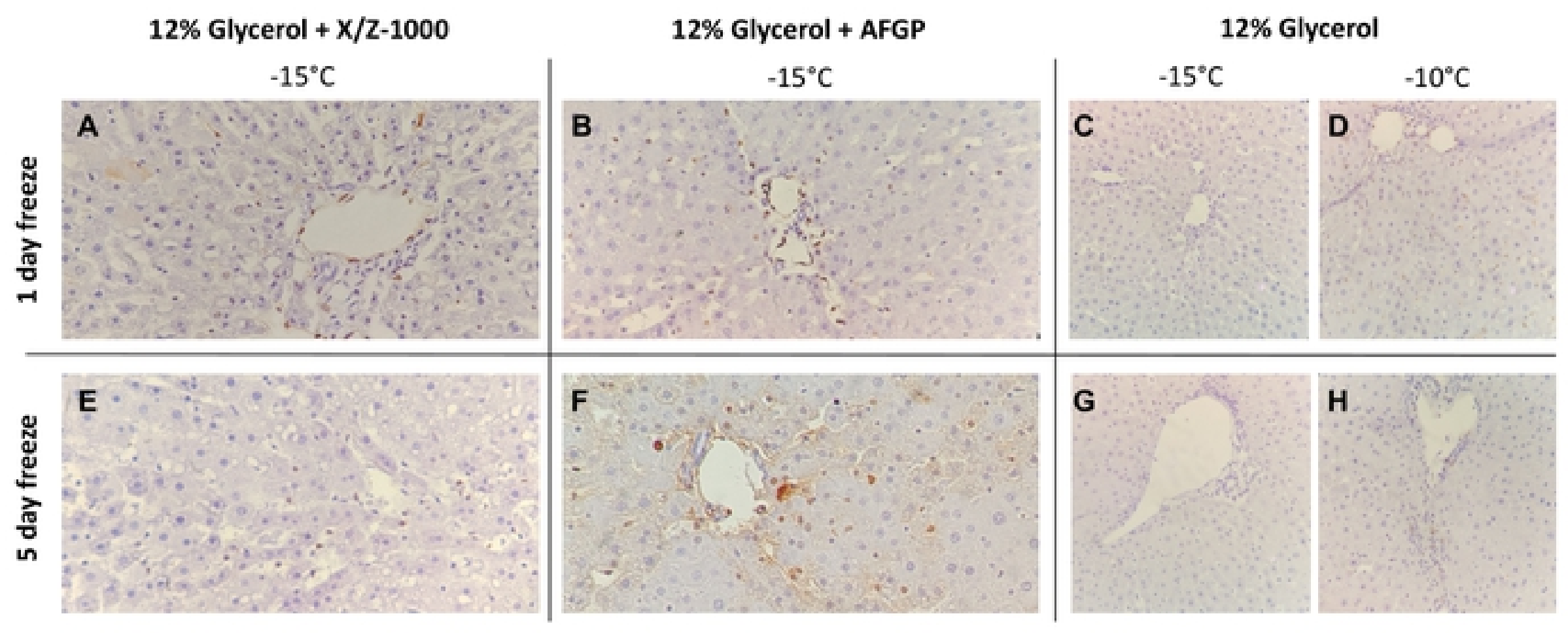

Figure 5: 40X Terminal deoxynucleotidyl transferase dUTP nick end labeling (TUNEL) staining

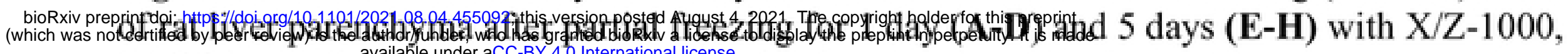
AFGP ice modulators versus $12 \%$ glycerol only. 